\title{
Information Fusion for Environmental Perception and Situation Awareness
}

\author{
Zheng Liu \\ Faculty of Applied Science, University of British Columbia \\ Canada
}

Fusion of information from multiple sources can benefit a variety of applications including battlefield situation awareness. The fusion operation has largely been performed with the data from physics-based sensors, such as radars, electrooptic (EO) camera, infrared (IR) imagers, etc. This fusion operation is also referred to as "hard fusion" and includes multimodal image fusion (e.g., EO and IR) as well as multi-perspective image fusion (e.g., multiple views of a single scene from two EO cameras). For instance, multi-sensor image fusion facilitates the scene perception through the representation and interpretation of the fused image at pixel-, feature-, and decision-levels. This talk will present the state-of-the-art for fusing multi-modal images with the results from the application of battlefield target detection.

Although hard fusion offers complementary information, the subtle connections and interactions between the entities for situation awareness are difficult to reveal with hard sensors. The exploitation of the fusion from physics-based sources and human-based sources has demonstrated significant advantage as richer content comes from human analysis, which is defined as a "soft" sensor information (as a human would write down in text information with more uncertainty in meaning). The fusion from physical sensors and human-based sources is also referred to as "hard" and "soft" fusion, or physics-based and human-based information fusion. This talk will also explore the potential of the "hard+soft" information fusion for the Level 2 fusion of situation assessment in the Data Fusion Information Group Model. 